\title{
Consumo de Gordura e Hipercolesterolemia em Uma Amostra Probabilística de Estudantes de Niterói, Rio de Janeiro
}

\begin{abstract}
RESUMO
Objetivo: Avaliar a associação entre consumo de gordura e colesterol total sérico (CT). Métodos: O inquérito foi realizado em uma amostra probabilística de adolescentes das escolas estaduais de Niterói, RJ. Foram obtidos dados antropométricos de 610 jovens, 577 participaram da coleta de sangue e 539 responderam o questionário de consumo alimentar. A análise multivariada de regressão linear incluiu como variáveis de confusão a hipercolesterolemia nos pais, idade e índice de massa corporal (IMC). Resultados: Concentrações aumentadas de CT (> $170 \mathrm{mg} / \mathrm{dL}$ ) ocorreram em $31,2 \%$ dos jovens. Ambos os sexos apresentaram freqüências elevadas (aproximadamente 45\%) de consumo inadequado de gorduras. O sobrepeso foi de 15,7\%, sem diferença estatisticamente significante entre os sexos $(p=0,83)$. Nenhum item alimentar isolado apresentou associação com o CT. IMC e presença de hipercolesterolemia nos pais mostraram associação estatisticamente significante com os teores de CT das meninas, e idade associou-se somente para os meninos. Verificou-se ausência de associação entre consumo de gordura e valores de CT. Conclusões: A combinação de altas taxas de hipercolesterolemia, sobrepeso e consumo de alimentos ricos em gordura sugerem que somente ações coletivas podem reduzir essas epidemias e que a redução na ingestão energética excessiva deve ser o principal objetivo destas ações. (Arq Bras Endocrinol Metab 2007;51/1:65-71)
\end{abstract}

Descritores: Hipercolesterolemia; Estudantes; Adolescente; Consumo de alimentos; Confiabilidade; Inquérito

\section{ABSTRACT}

Fat Intake and Hypercholesterolemia in a Probabilistic Sample of Adolescents From Niterói, Rio de Janeiro.

Objective: To evaluate the relationship between fat intake and serum cholesterol. Methods: A survey was conducted in a probabilistic sample of students from public schools in Niterói, state of Rio de Janeiro, Brazil. Anthropometric data were collected on 610 youngsters, 573 of them had blood drown, and 539 answered a simplified questionnaire on fat intake. Multivariate linear regression included as confounders: parents' cholesterolemia, adolescent age and body mass index (BMI). Results: The prevalence of high concentrations (> $170 \mathrm{mg} / \mathrm{dL}$ ) of serum cholesterol was $31.2 \%$. Both sexes presented high frequencies (45\% approximately) of inadequate intake of fatty foods. Prevalence of overweight was $15.7 \%$, without significant statistical difference between sexes $(p=0.83)$. Total fat intake as measured by the questionnaire and each one of the nine high fat items was not associated with youngsters' cholesterol. Among girls BMI and parents' hypercholesterolemia were associated with cholesterol levels, but for boys only age showed statistically significant association. Lack of association between fat intake and cholesterol levels was observed. Conclusion: The combined higher prevalence of hypercholesterolemia, overweight and intake of high fat foods suggest that only public health action may curb these epidemics. This may indicate that overall energy intake should be the target of the actions. (Arq Bras Endocrinol Metab 2007;51/1:65-71)

Keywords: Hypercholesterolemia; Student; Adolescent; Food consumption; Survey

\section{artigo original}

\author{
MÁRCIA H. TEIXEIRA \\ GLÓRIA V. DA VEIGA \\ ROSELY SICHIERI
}

Instituto de Medicina Social da Universidade do Estado do Rio de Janeiro (MHT \& RS) e Instituto de Nutrição Josué de Castro da Universidade Federal do Rio de Janeiro (GVV), RJ.

Recebido em 16/11/05

Revisado em 28/04/06

Aceito em 28/08/06 
A LTERAÇÕES DO PERFIL LIPÊMICO, presentes na infância e adolescência, persistem na idade adulta, constituindo um importante fator de risco para o desenvolvimento das lesões ateroscleróticas, como demonstrado em um estudo de seguimento para doenças cardiovasculares em crianças e jovens americanos. Aproximadamente 50\% dessas crianças apresentavam colesterol total (CT) elevado e baixos valores de lipoproteína de alta densidade (HDL), que se mantiveram 10 a 15 anos mais tarde (1).

Das variáveis ambientais envolvidas na determinação do perfil lipêmico, a dieta é considerada uma das mais importantes, particularmente pela sua relação com as doenças coronarianas (2). Os fatores dietéticos que exercem efeitos negativos sobre o perfil lipêmico são a alta ingestão de gordura saturada e de colesterol que estão associados a valores séricos elevados de CT e $\operatorname{LDL}(2)$.

Block e cols. (3) desenvolveram um questionário para avaliação do consumo de gordura com ênfase na gordura saturada, e um questionário simplificado para auto-avaliação em adolescentes de consumo de alimentos com alto teor de gordura e ácidos graxos trans foi desenvolvido a partir de dados de um questionário semiquantitativo de freqüência de consumo alimentar (QFCA). Dos 80 itens iniciais, nove itens explicaram $85 \%$ da estimativa da variação do colesterol sérico em função da dieta, e esse questionário (4) foi aplicado no presente estudo, cujo objetivo foi verificar a associação entre consumo alimentar e valores de colesterol sérico.

\section{MATERIAL E MÉTODOS}

Uma amostra probabilística dos adolescentes na faixa etária de 12 a 19 anos, estudantes da $5^{\text {a }}$ série do ensino fundamental a $3^{\text {a }}$ série do ensino médio da rede de ensino estadual, na cidade de Niterói, RJ, foi avaliada em 2003. Foram excluídos os adolescentes portadores de deficiência física que impedisse a avaliação antropométrica e adolescentes grávidas.

O cálculo da amostragem foi feito levando em consideração uma prevalência de $25 \%$ de hipercolesterolemia (5), um intervalo de confiança de $95 \%$ e precisão de $5 \%$. A amostragem por conglomerado (turmas) totalizaria 600 estudantes (6). Prevendo perda de 30\%, segundo estudo piloto realizado, a amostra final foi estimada em 780 adolescentes, equivalente a 26 turmas de 30 alunos, da $5^{\text {a }}$ série do ensino fundamental ao $3^{\circ}$ ano do ensino médio. Foram sorteadas 28, prevendo-se turmas com número de alunos inferior a 30. Das turmas sorteadas, todos os alunos que atendiam aos critérios de elegibilidade foram convidados a participar do estudo, totalizando 757 . Obtiveram-se dados antropométricos de 610 adolescentes (43 não obtiveram autorização dos pais, 85 não quiseram participar e 19 não compareceram nos dias da coleta). Destes, 577 participaram da coleta de sangue (23,7\% de não resposta) e 539 responderam o questionário de consumo. Seis relataram ter feito uso de medicamentos para hipercolesterolemia, sendo incluídos como caso de hipercolesterolemia.

Foram obtidas medidas de peso e estatura, sendo o peso aferido em balança eletrônica e portátil com capacidade até $150 \mathrm{~kg}$ e variação de $50 \mathrm{~g}$, estando os adolescentes sem calçados, usando roupas leves e sem portar objetos pesados.

Para a aferição da estatura, foi usado antropômetro portátil com extensão de $200 \mathrm{~cm}$ e escala de $0,1 \mathrm{~cm}$. A estatura foi medida com os estudantes descalços, com os calcanhares juntos e encostados ao antropômetro, e com a cabeça no plano horizontal de Frankfurt. Foram realizadas duas mensurações, tomando-se a média como estimativa da estatura. A variação admitida entre as duas mensurações foi $0,5 \mathrm{~cm}$. Se ultrapassasse este valor, as duas medidas eram anuladas e repetidas.

Com os dados de peso e estatura calculou-se o índice de massa corporal - IMC (peso/estatura ${ }^{2}$ ). O sobrepeso foi classificado segundo proposta de Cole e cols. (7), agrupando-se as categorias sobrepeso e obesidade, dada a baixa prevalência de obesidade encontrada (2,7\%). Foram considerados com magreza os adolescentes que estavam abaixo do percentil 5 da referência de Must e cols. (8), e eutróficos aqueles que ficaram igual ou acima do percentil 5 e abaixo dos valores estabelecidos por Cole e cols. (7) para sobrepeso.

Para a avaliação bioquímica foi coletada amostra de aproximadamente $10 \mathrm{~mL}$ de sangue, por laboratorista qualificado, no período da manhã e com os adolescentes em jejum de 12 horas. O sangue colhido era armazenado em isopor com gelo seco e levado, imediatamente após a coleta, para o laboratório de análises clínicas, onde foi centrifugado e analisado. O colesterol sérico foi avaliado pelo método enzimático automatizado.

O questionário simplificado com nove itens, proposto por Chiara \& Sichieri (4), foi auto-preenchido pelos adolescentes durante o período de aula. A informação sobre história familiar de hipercolesterolemia foi obtida com os responsáveis dos alunos.

O desenvolvimento pubertário foi avaliado através da auto-avaliação dos estágios de maturação sexual descritos por Tanner (9), procedimento previamente validado (10). Foram apresentadas aos jovens, de forma individual e reservada, as pranchas elaboradas pelo Ministério da Saúde contendo desenhos dos estágios de maturação sexual. Para as meninas foi também obtida informação sobre a presença da menarca e idade de ocorrência da mesma.

Para o controle de qualidade no que diz respeito aos dados faltantes e inconsistentes, os questionários eram 
checados logo após a entrega. Caso houvesse algum erro que pudesse ser corrigido no momento, assim era feito, e caso o erro fosse proveniente de informações incompreensíveis, provenientes do responsável, era solicitado esclarecimento por consulta telefônica. Eram realizadas duas tentativas de contato com o adolescente ou com o responsável por telefone.

Os dados coletados foram duplamente digitados através do programa EPI-INFO versão 6.04. (11). Foram estabelecidos mecanismos de restrição de entradas de dados de maneira a evitar possíveis erros de digitação.

Os pontos de corte utilizados para definição de colesterolemia nos jovens foram os estabelecidos pelo National Cholesterol Education Program Expert Panel on Blood Cholesterol levels in children and adolescents (12).

O questionário alimentar simplificado (QAS) foi avaliado segundo os escores propostos pelos autores (4) quanto ao risco cardiovascular, classificando o consumo em adequado (100 pontos), elevado (entre 101 e 119 pontos) e excessivo (igual ou maior que 120 pontos). Também foi analisada a soma da freqüência de consumo dos nove itens do QAS sem considerar os escores propostos.

Estimou-se o efeito do desenho do estudo comparando-se o erro padrão obtido usando o procedimento estatístico que levou em consideração o efeito de conglomerado e a expansão dos dados com o erro padrão obtido sem considerar o desenho do estudo.

Utilizou-se o teste " $t$ " de Student ou análise de variância para a comparação estatística das diferenças entre as médias e o teste qui-quadrado para as freqüências das variáveis categóricas. A análise multivariada foi realizada através da regressão linear.

Dado que o sexo (13), idade (13), IMC (14,15), estágios de maturação sexual (13) e história familiar de hipercolesterolemia (12) associam-se a hipercolesterolemia, estes foram avaliados como variáveis de confusão.

Para avaliação do grau de maturação sexual dos adolescentes foi utilizada a idade da menarca e os critérios de Tanner (9) para o desenvolvimento mamário nas meninas e a genitália para os meninos (16).

Para a análise dos dados levou-se em consideração a expansão da amostra (calculando-se o peso de cada indivíduo através de $1 / \mathrm{P}$, onde $\mathrm{P}=$ probabilidade de cada aluno ter participado da pesquisa) e o efeito do desenho da amostra por conglomerado (turmas), utilizando-se o comando Proc Surveymeans, do pacote Statistical Analysis System, versão 8.2 (SAS, Institute Inc, Cary, NC, USA) (17).

A variável colesterol sérico foi normalizada através de transformação logarítmica.

O protocolo do estudo foi aprovado pelo Comitê de Ética em Pesquisa do Hospital Universitário Clementino Fraga Filho da Universidade Federal do Rio de Janeiro. Os resultados da avaliação antropométrica e bioquímica foram entregues aos alunos, e aqueles que apresentaram alterações consideradas de risco receberam orientações.

\section{RESULTADOS}

A média de colesterol sérico foi significantemente mais elevada para o sexo feminino. História de hipercolesterolemia nos pais foi referida por $23 \%$ dos jovens, e grande parte dos adolescentes classificaram-se nos estágios 4 e 5 de maturação sexual, sendo que somente seis meninas não referiram menarca (tabela 1 ).

Do total de jovens, $31,2 \%$ apresentaram concentrações de CT sérico acima dos valores limítrofes (> $170 \mathrm{mg} / \mathrm{dL}$ ) e ambos os sexos apresentaram freqüências elevadas de consumo inadequado de gorduras, segundo Chiara \& Sichieri (4) (tabela 2).

Mais recentemente foram recomendados pontos de corte mais restritivos para colesterolemia em adolescentes (18). Utilizando-se estes novos pontos de corte de CT sérico, as prevalências de valores limítrofes ( 150 a $169 \mathrm{mg} / \mathrm{dL}$ ) foram de $24 \%$ nos meninos e $23 \%$ nas meninas, e de CT sérico aumentado (> $170 \mathrm{mg} / \mathrm{dL}$ ) foram de $26 \%$ e $33 \%$, respectivamente, para jovens do sexo masculino e feminino.

O sobrepeso foi observado em 15,7\%, sem diferença estatisticamente significante entre os sexos $(\mathrm{p}=0,83)$.

Os alimentos mais consumidos diariamente pelos jovens foram manteiga/margarina, biscoito e leite, sendo que biscoito é um dos itens mais consumidos (figura 1). A freqüência no consumo de leite foi maior no sexo masculino $(\mathrm{p}=0,02)$. Para os outros itens alimentares não houve diferença estatisticamente significante quanto ao consumo entre os sexos.

As médias de colesterol sérico associaram-se à idade, para ambos os sexos. Para as meninas, associaramse também ao estado nutricional e a história de hipercolesterolemia nos pais (tabela 3 ).

A concentração de colesterol sérico e a pontuação do questionário de consumo foram pouco correlacionadas, seja com o uso do escore, proposto pelo questionário $(\mathrm{r}$ de Spearman $=-0,04$ e $\mathrm{p}=0,33$ ), ou utilizando-se a soma da freqüência de consumo diário de todos os nove itens $(\mathrm{r}$ de Spearman $=-0,07 \mathrm{e}$ $\mathrm{p}=0,08)$. Mesmo com o ajuste para possíveis variáveis de confusão, através de três modelos de regressão linear, o consumo não associou-se aos níveis de colesterol (tabela 4). Foram testados também os mesmos modelos utilizando como variável dependente o colesterol sérico subtraído da fração HDL, porém não houve diferença nos resultados encontrados em relação aos modelos anteriores (dados não mostrados).

Nos modelos 1 e 2 foram incluídas as variáveis estatisticamente significantes na análise bivariada $(\mathrm{p} \leq$ $0,05)$. No modelo 1 utilizou-se a soma da freqüência 
Tabela 1. Médias, erros-padrão e freqüências das variáveis estudadas, segundo sexo, em uma amostra probabilística de adolescentes de escolas estaduais de Niterói, RJ, 2003.

\begin{tabular}{|c|c|c|}
\hline & Masculino $(n=221)$ & Feminino $(n=383)$ \\
\hline Idade (anos) & $16,5(0,3)$ & $16,1(0,3)$ \\
\hline Média (EP) & $(\%)$ & $(\%)$ \\
\hline $12-15$ & 20,4 & 32,4 \\
\hline $16-17$ & 48,4 & 43,6 \\
\hline $18-19$ & 31,2 & 24,0 \\
\hline \multicolumn{3}{|l|}{$\mathrm{IMC}^{*}\left(\mathrm{~kg} / \mathrm{m}^{2}\right)$} \\
\hline Média (EP) & $21,1(0,3)$ & $21,1(0,2)$ \\
\hline \multicolumn{3}{|l|}{ Colesterol sérico (mg/dL) } \\
\hline Média (EP) & $150,5(4,1)$ & $160,4(3,50)^{1}$ \\
\hline Diagnóstico prévio de hipercolesterolemia** (\%) & 1,83 & 4,8 \\
\hline \multicolumn{3}{|l|}{ História familiar de hipercolesterolemia*** $(\%)$} \\
\hline Pai ou mãe & 20,0 & 21,2 \\
\hline Ambos & 2,0 & 3,1 \\
\hline Idade da menarca (anos) $)^{* * *}$ & & $12,0(0,07)$ \\
\hline Média (EP) & - & $(\%)$ \\
\hline $7-10$ & - & 8,9 \\
\hline $11-12$ & - & 58,8 \\
\hline $13-17$ & - & 32,3 \\
\hline Estágios de Tanner & $(\%)$ & $(\%)$ \\
\hline $2-3$ & 29,9 & 37,8 \\
\hline 4 & 57,0 & 39,8 \\
\hline 5 & 13,1 & 22,5 \\
\hline
\end{tabular}

Tabela 2. Prevalência expandida de hipercolesterolemia* e de consumo inadequado de gorduras**, segundo sexo, em uma amostra probabilística de adolescentes de escolas estaduais de Niterói, RJ, 2003.

\begin{tabular}{|c|c|c|c|c|c|c|}
\hline & \multicolumn{3}{|c|}{ Masculino } & \multicolumn{3}{|c|}{ Feminino } \\
\hline & $\mathbf{N}$ & $\%$ & IC $95 \%$ & $\mathbf{N}$ & $\%$ & IC $95 \%$ \\
\hline \multicolumn{7}{|l|}{ Colesterol sérico (mg/dL) } \\
\hline 170-199 (limítrofe) & 44 & 18,4 & $12,76-24,00$ & 99 & 23,6 & $17,60-29,60$ \\
\hline$\geq 200$ (elevado) & 20 & 7,9 & $2,75-13,04$ & 48 & 9,8 & $5,33-14,34$ \\
\hline \multicolumn{7}{|l|}{ Consumo alimentar } \\
\hline Elevado & 23 & 8,9 & $4,52-13,44$ & 40 & 9,4 & $5,40-13,44$ \\
\hline Excessivo & 75 & 34,0 & $27,36-40,61$ & 130 & 35,8 & $30,32-41,24$ \\
\hline
\end{tabular}

* Classificação para colesterol sérico em mg/dL estabelecidos pelo National Cholesterol Education Program (12); ** Consumo segundo questionário simplificado (4). Elevado: entre 101 e 119 pontos, e Excessivo: maior ou igual a 120 pontos.

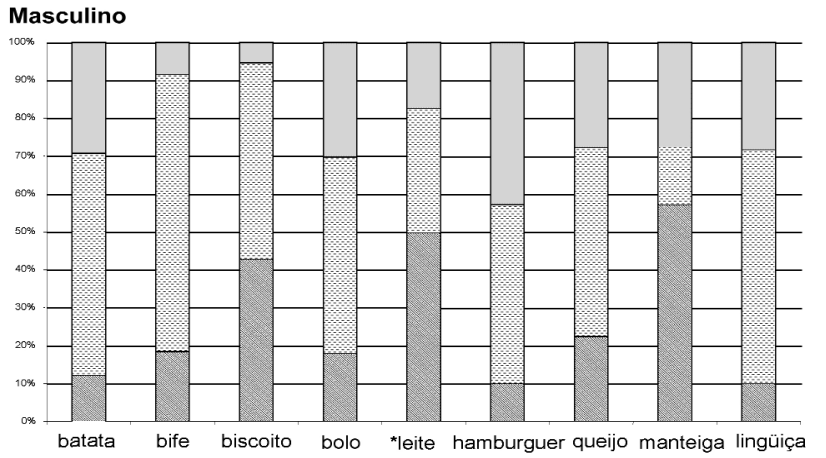

Qui-quadrado: ${ }^{*} \mathrm{p}=0,02$

\begin{abstract}
Diário
\end{abstract}

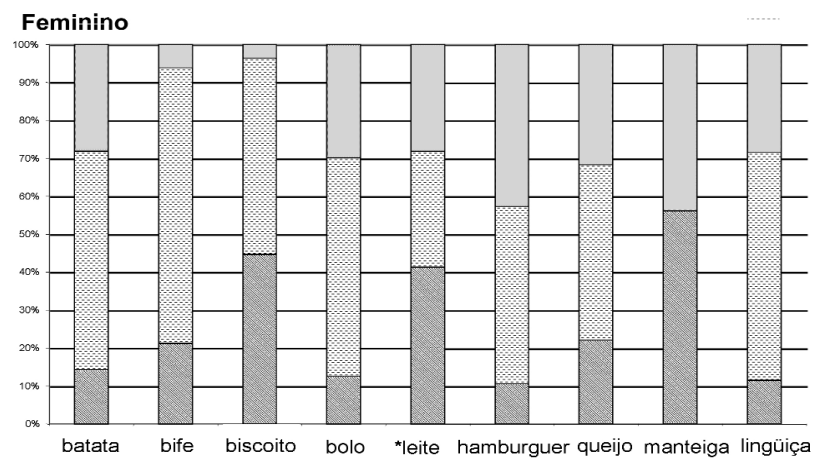

$\square$ Nunca / quase nunca

Figura 1. Consumo de alimentos segundo sexo, em uma amostra probabilística de adolescentes de escolas estaduais de Niterói, RJ, 2003. 
Tabela 3. Médias expandidas ( $\mathrm{mg} / \mathrm{dL}$ ) e desvios-padrão (DP) de colesterol total sérico, segundo idade, estado nutricional, história de hipercolesterolemia nos pais e maturação sexual, em uma amostra probabilística de adolescentes de escolas estaduais de Niterói, RJ, 2003.

\begin{tabular}{|c|c|c|c|c|c|c|}
\hline & \multicolumn{6}{|c|}{ Colesterol total sérico (mg/dL) } \\
\hline & \multicolumn{3}{|c|}{ Masculino } & \multicolumn{3}{|c|}{ Feminino } \\
\hline & $\mathbf{N}$ & Média & DP & $\mathbf{N}$ & Média & DP \\
\hline \multicolumn{7}{|c|}{ Idade (anos) } \\
\hline $12-15$ & 42 & 141,0 & 32,3 & 114 & 153,7 & 31,1 \\
\hline $16-17$ & 104 & 153,7 & 33,7 & 162 & 170,9 & 37,5 \\
\hline $18-19$ & 63 & 157,0 & 33,7 & 86 & 160,4 & 33,8 \\
\hline$p^{*}$ & & 0,047 & & & 0,0003 & \\
\hline \multicolumn{7}{|c|}{ Estado nutricional } \\
\hline Eutrófico & 167 & 151,6 & 32,6 & 299 & 161,1 & 34,3 \\
\hline Sobrepeso & 35 & 155,2 & 38,2 & 57 & 174,1 & 40,6 \\
\hline Magreza & 7 & 149,8 & 41,2 & 6 & 149,0 & 14,6 \\
\hline$p^{*}$ & & 0,835 & & & 0,024 & \\
\hline \multicolumn{7}{|c|}{ Hipercolesterolemia nos pais } \\
\hline Ausente & 151 & 150,7 & 33,7 & 256 & 159,8 & 34,0 \\
\hline Um deles & 38 & 155,0 & 37,8 & 72 & 171,2 & 38,0 \\
\hline Ambos & 4 & 181,2 & 15,7 & 11 & 187,0 & 41,6 \\
\hline$p^{*}$ & & 0,187 & & & 0,004 & \\
\hline \multicolumn{7}{|c|}{ Maturação Sexual** } \\
\hline 2 e 3 & 64 & 153,8 & 38,8 & 134 & 158,4 & 33,6 \\
\hline 4 & 117 & 151,2 & 31,3 & 146 & 164,2 & 34,8 \\
\hline 5 & 28 & 152,4 & 32,1 & 82 & 168,3 & 38,7 \\
\hline$p^{*}$ & & 0,882 & & & 0,115 & \\
\hline
\end{tabular}

* p: ANOVA; ** genitália e mamas segundo estágios de Tanner (9).

Tabela 4. Regressão linear multivariada para avaliar associação do colesterol sérico com medidas de consumo alimentar e com os itens do questionário simplificado, segundo sexo, em uma amostra probabilística de adolescentes de escolas estaduais de Niterói, RJ, 2003.

\begin{tabular}{|c|c|c|c|c|c|c|}
\hline & \multicolumn{3}{|c|}{ Masculino } & \multicolumn{3}{|c|}{ Feminino } \\
\hline & $\beta$ & p-valor & $\mathbf{R}^{2}$ ajustado & $\beta$ & p-valor & $\mathbf{R}^{2}$ ajustado \\
\hline \multicolumn{7}{|l|}{ Modelo 1} \\
\hline Soma das freqüência dos nove itens do QAS & $-1,56$ & 0,018 & 0,09 & $-0,25$ & 0,56 & 0,13 \\
\hline Idade (anos) & 9,24 & 0,016 & & 4,24 & 0,15 & \\
\hline Hipercolesterolemia nos pais & 5,81 & 0,502 & & 14,14 & 0,001 & \\
\hline $\mathrm{IMC}^{1}\left(\mathrm{~kg} / \mathrm{m}^{2}\right)$ & 1,10 & 0,080 & & 1,60 & 0,010 & \\
\hline Desenvolvimento mamário* & - & - & - & 4,96 & 0,20 & \\
\hline \multicolumn{7}{|l|}{ Modelo 2} \\
\hline Avaliação de risco da dieta segundo QAS** & $-0,05$ & 0,06 & 0,08 & $-0,004$ & 0,86 & 0,13 \\
\hline Idade (anos) & 9,51 & 0,01 & & 4,31 & 0,15 & \\
\hline Hipercolesterolemia nos pais & 5,54 & 0,52 & & 14,01 & 0,0001 & \\
\hline $\mathrm{IMC}^{1}\left(\mathrm{~kg} / \mathrm{m}^{2}\right)$ & 1,08 & 0,09 & & 1,60 & 0,009 & \\
\hline Desenvolvimento mamário* & - & - & - & 4,86 & 0,21 & \\
\hline \multicolumn{7}{|l|}{ Modelo $3^{* * *}$} \\
\hline & $\beta$ & p-valor & $\beta$ & p-valor & & \\
\hline Batata & $-5,09$ & 0,30 & $-1,39$ & 0,60 & & \\
\hline Bife & $-3,88$ & 0,12 & $-0,74$ & 0,82 & & \\
\hline Biscoito & $-3,25$ & 0,22 & $-0,86$ & 0,69 & & \\
\hline Bolo & 1,17 & 0,78 & $-3,18$ & 0,18 & & \\
\hline Leite & $-5,34$ & 0,04 & 0,41 & 0,82 & & \\
\hline Hambúrguer & $-1,85$ & 0,68 & 6,36 & 0,07 & & \\
\hline Queijo & 0,20 & 0,95 & $-1,06$ & 0,53 & & \\
\hline Manteiga/ margarina & $-5,59$ & 0,07 & $-0,38$ & 0,83 & & \\
\hline Lingüiça & $-6,92$ & 0,13 & $-4,29$ & 0,18 & & \\
\hline
\end{tabular}

* Segundo estágios de Tanner (9); ** QAS - Questionário alimentar simplificado (4); ** Ajustado por IMC1 (índice de massa corporal), idade, história de hipercolesterolemia nos pais e desenvolvimento mamário (sexo feminino). 
dos nove itens do questionário, sem levar em consideração os escores propostos para o mesmo, e no modelo 2 usou-se a pontuação proposta pelo questionário para avaliar o consumo alimentar. IMC e história de hipercolesterolemia nos pais associaram-se às concentrações séricas de colesterol para as meninas, enquanto que idade manteve-se associada somente para os meninos, em ambos os modelos. No modelo 3 evidenciase, também, que nenhum item alimentar isolado apresentou associação estatisticamente significante com o colesterol sérico dos jovens de ambos os sexos, mesmo quando o modelo foi ajustado por idade, história de hipercolesterolemia dos pais, índice de massa corporal e desenvolvimento mamário (tabela 4).

\section{DISCUSSÃo}

O excesso de peso é um fator importante para a hipercolesterolemia. A freqüência de sobrepeso no grupo estudado foi de aproximadamente $16 \%$, valor próximo aos encontrados para o Brasil $(14,15,19)$.

Contudo, somente em meninas encontrou-se associação entre estado nutricional e valores de colesterol sérico. A associação positiva do colesterol sérico com o IMC foi também observada em outros estudos $(14,15)$. A ausência de associação entre estado nutricional e teores de colesterol sérico nos meninos verificada no presente estudo não é explicada pelo consumo alimentar, uma vez que a única diferença observada foi uma menor ingestão de leite entre as meninas.

Um alto IMC em meninos é menos associado com adiposidade (20), o que poderia explicar os achados. A freqüência das concentrações aumentadas de colesterol foi alta $(31,2 \%)$, conforme registrado também em outros estudos nacionais $(5,15)$.

Moura e cols. (13) observaram, em estudo com escolares de 7 a 14 anos, residentes em Campinas, que meninas apresentavam pico de colesterol sérico aos 8 anos, queda até os 12 anos, novo pico aos 13 anos e diminuição aos 14 anos, enquanto que para os meninos, valores crescentes ocorriam até os 11 anos, com decréscimo a partir dessa idade. No presente estudo, a média de colesterol sérico também foi mais elevada no sexo feminino, aumentando com a maturação sexual.

Dos alimentos marcadores para consumo de gordura, o que apresentou maior freqüência de consumo pelos jovens foi a manteiga/margarina. O consumo do leite foi significantemente diferente entre meninos e meninas, e somente $50 \%$ dos meninos o consomem diariamente. Para as meninas, essa ingestão é ainda menor. Essa situação não difere muito em relação a outros estudos brasileiros. Lerner e cols. (21), avaliando a ingestão de cálcio de adolescentes de escolas públicas de Osasco, verificaram que as meninas ingerem cerca de $190 \mathrm{~mL}$ de leite por dia, enquanto os meninos bebem $240 \mathrm{~mL}$, ambos com consumo muito abaixo das recomendações. Andrade e cols. (22) verificaram, em uma amostra probabilística do Município do Rio de Janeiro, que meninas tinham consumo de leite e derivados abaixo das recomendações diárias, e este consumo era ainda menor para aquelas que apresentavam sobrepeso, o que não ocorria com os meninos, que tinham consumo similar independente do estado nutricional.

Por outro lado, o consumo de alimentos marcadores de gorduras foi elevado. Aproximadamente 45\% dos adolescentes classificaram-se como tendo consumo elevado ou excessivo, semelhante ao que tem sido relatado na literatura (15).

No presente estudo, a concentração de colesterol sérico associou-se com o IMC e história de hipercolesterolemia nos pais, somente nas meninas. Para ambos os sexos, tanto a proposta de pontuação baseada em modelo de regressão (4) quanto a freqüência de consumo não mostraram associação, havendo, inclusive, tendência de associação inversa entre consumo e concentração sérica de colesterol, particularmente para o leite e manteiga/margarina.

Verificou-se a ausência de associação entre consumo de gordura e valores de CT. A combinação de altas taxas de hipercolesterolemia, sobrepeso e consumo de alimentos ricos em gordura sugerem que somente ações coletivas podem reduzir estes riscos à saúde dos adolescentes, e que a ingestão energética excessiva deve ser o principal objetivo destas ações.

\section{REFERÊNCIAS}

1. Webber LS, Srinivasan SR, Wattigney WA, Berenson GS. Tracking of serum lipids and lipoproteins from childhood to adulthood. Am J Epidemiol 1991;133:884-99.

2. World Health Organization. Diet, Nutrition and the Prevention of Chronic Diseases. Geneva, WHO Technical Report Series, n.916;2003.

3. Block G, Cliford C, Naughlton MD, Henderson M, McAdams M. A brief dietary screen for high fat intake. J Nutr Educ 1989;21:199-207.

4. Chiara VL, Sichieri R. Food consumption of adolescents. A simplified questionnaire for evaluating cardiovascular risk. Arq Bras Cardiol 2001;77:337-41.

5. Gerber ZRS, Zielinsky P. Fatores de risco de aterosclerose na infância: um estudo epidemiológico. Arq Bras Cardiol 1997;69:231-6.

6. Lwanga SK, Lemeshow S. Sample size determination in health studies: a practical manual. World Health Organization, Geneva;1991. 
7. Cole JT, Bellizi CM, Flegal MK, Dietzs HW. Establishing a standard definition for child overweight and obesity worldwide: international survey. BMJ 2000;320:1240-3.

8. Must A, Dallal GE, Dietz WH. Reference data for obesity: 85th and 95th percentiles of body mass index $\left(\mathrm{wt} / \mathrm{ht}^{2}\right)$ and triceps skinfold thickness. Am J Clin Nutr 1991;53:839-46.

9. Tanner JM. Growth at adolescence. Oxford: Blackwell;1962.

10. Saito MI Maturação sexual: auto-avaliação do adolescente. Pediatrics 1984;6:111-5.

11. Dean AG, Dean JA, Coulombier D. Epi Info 6.04- A Word Processing database and Statistics Program for Public Health on IBM-Compatibles microcomputers. Atlanta: Center for Disease Control and Prevention/ World Health Organization, 1996.

12. National Cholesterol Education Program. Expert panel on blood cholesterol levels in children and adolescents. Pediatrics 1992;89(3 Suppl):525-84.

13. Moura EC, Castro CM, Mellin AS, Figueiredo DB. Perfil lipídico em escolares de Campinas, SP, Brasil. Rev Saúde Pública 2000;34:499-505.

14. Coronelli CLS, Moura EC. Hipercolesterolemia em escolares e seus fatores de risco. Rev Saúde Pública 2003;37:24-31.

15. Rabelo LM, Viana RM, Schimith MA, Patin RV, Valverde MA, Denadai RC, et al. Fatores de risco para doença aterosclerótica em estudantes de uma universidade privada em São Paulo. Arq Bras Cardiol 1999;72:569-74.

16. Coelho $\mathrm{KSC}$, Sichieri $\mathrm{R}$, Ganzalez $\mathrm{CH}$. Influencia de la maturacion sexual em el índice de masa corporal em adolescentes de bajo nível socioeconômico de Rio de Janeiro. Rev Chil Nutr 2002;29:33-9.
17. Sas. The Statistical Analysis System. SAS Institute Inc. Cary. USA, 1998.

18. I Diretriz de Prevenção da Aterosclerose na infância e na adolescência. Sociedade Brasileira de Cardiologia. Arq Bras Cardiol 2005;85(6 Suppl):3-36.

19. Veiga GV, Cunha AS, Sichieri R. Trends in overweight among adolescents living in the poorest and richest regions of Brazil. Am J Public Health 2004;94:1544-8.

20. Veiga GV, Dias PC, Anjos LA. Comparison of American and Brazilian BMI distribution curves in the assessment of overweight and obesity in a sample of middle-class Brazilian adolescents. Rev Panam Salud Pública 2001;10:79-85.

21. Lerner BR, Lei DLM, Chaves SP, Freire RD. O cálcio consumido por adolescentes de escol escolas as públicas de Osasco, São Paulo. Rev Nutr 2000;13:57-63.

22. Andrade RG, Pereira RA, Sichieri R. Consumo alimentar de adolescentes com e sem sobrepeso do Município do Rio de Janeiro. Cad Saúde Pública 2003;19:1485-95.

Endereço para correspondência:

Márcia Henriques Teixeira

Rua Fortaleza 140

21210-490 Rio de Janeiro, RJ

E-mail: marciaht@click21.com.br 